\title{
A CASE OF DISPROPORTIONATE MACRODACTYLY OR A MILD FORM OF PROTEUS SYNDROME? AN INTERESTING CASE
}

\author{
Shalimar Abdullah ${ }^{1}$, Nor Hazla Mohd Haflah ${ }^{1}$, Jamari Sapuan ${ }^{1}$, Srijit Das ${ }^{2}$ \\ National University of Malaysia, Malaysia Medical Centre (UKMMC), Malaysia: Department of Orthopaedics ${ }^{\mathbf{1}}$, \\ Department of Anatomy ${ }^{2}$
}

\begin{abstract}
Summary: We present a 20-year-old Malay male whom we believe has Proteus syndrome, a rare congenital disorder of asymmetrical overgrowth of body tissues. There are fewer than 100 confirmed cases reported worldwide thus the clinical presentation and histopathological findings are of significance. Our patient presented with an overgrown right small finger and subcutaneous purplish pigmentation over his left upper arm and chest since birth. His small finger gradually increased in size. He had no abnormalities in sensation or power. Radiographs revealed a delta shaped middle phalanx of the small finger. His activities of daily living were uninterrupted but he requested debulking surgery for cosmetic reasons. Histopathological examination reported hypertrophic fatty tissue composed of well formed lobules of mature adipocytes interspersed with fibrous elements.
\end{abstract}

Key words: Proteus syndrome; Finger; Fatty tissue; Hypertrophy; Skin; Pigmentation

\section{Introduction}

In the year 1983, Wiedemann and co researchers reported four cases of children with partial gigantism, hemihypertrohy, subcutaneous tumours, macrocephaly or skull anomalies (11). The name of Proteus syndrome was given to this new interesting phenotyping polymorphism. In fact, the name Proteus syndrome (PS) was derived from the name of a Greek God who could change his shape according to his wish (Proteus, gr.= the polymorphous) (6). The genetic basis of this complex hamartomatous disorder is currently unknown (6). The famous patient, Joseph Merrick or "the elephant man" was thought to suffer from Proteus syndrome.

PS is considered to be a rare, complex and highly variable sporadic disorder which is responsible for asymmetrical overgrowth of multiple tissues of the body $(1,9)$. Its variability in tissue involvement may even present with variable clinical presentations which makes its diagnosis more difficult.

PS is thought to be very rare with less than 100 confirmed affected individuals reported worldwide (2). Thus, the clinical facts and the histopathological findings assume great significance.

\section{Case Report}

A 20-year-old Malay man attended the Orthopedics department of the Universiti Kebangsaan Malaysia Medical
Centre (UKMMC) with a disproportionately large right small finger (Fig. 1A,B,C). Prior consent was obtained in the consent form before proceeding with further research findings.

He complained that his right small finger was slightly large at birth and gradually increased in size over the years. However, it did not interfere with his daily activities. He was able to ride a motorbike gripping the handle with all his digits except the small finger which he kept in extension as it was too bulky to be in a flexed position. He had a subcutaneous purplish pigmentation over his left upper chest and arm (Fig. 2A). He did not have any subcutaneous nodules to suggest the presence of von Recklinghausen's disease.

Physical examination revealed a macrodactylous right little finger deviated radially. Sensation was intact with a 2point discrimination of $6 \mathrm{~mm}$ on either side of the digit. The finger was lobulated and soft in consistency. Flexion was limited at the metacarpophalangeal, distal and proximal interphalangeal joint due to bulky soft tissue. Motor and sensory examination of the ulnar nerves were normal.

Radiographs revealed a middle phalanx which appeared slightly delta in shape and the distal interphalangeal joint was opened up on the ulnar side. The entire digit was deviated radially at an angle of 50 degrees (Fig. 2B).

Intraoperatively, the whole digit was composed of hypertrophic fatty tissue encircling the neurovascular bundle. The neurovascular bundle size was slightly enlarged and difficult to differentiate from the surrounding fat. The flexor tendon was normal. Post-operatively, he had stiffness and 
numbness with an inflamed wound. As he lived out-of-town (about 2 hours drive from our hospital), we extended his ward stay by 1 week to ensure the inflamed wound settled down and for aggressive daily hand physiotherapy in ward. He was subsequently referred to the general physiotherapist in the small district hospital in his area (they had no hand therapist). On his first follow-up, 4 weeks post-operation, he only had residual stiffness (Fig 1D).

\section{Histopathological findings}

Multiple fragments of fibro-fatty tissue, measuring 10 $\mathrm{cm}$ in aggregate were sent to the histopathological laboratory. Microscopically, the fatty tissue was composed of well formed lobules of mature adipocytes interspersed with fibrous connective tissue elements within which there were vessels and nerves. No lipoblast or mitotic figures were seen and the diagnosis was given as lipohamartoma (Fig. 3A,B).
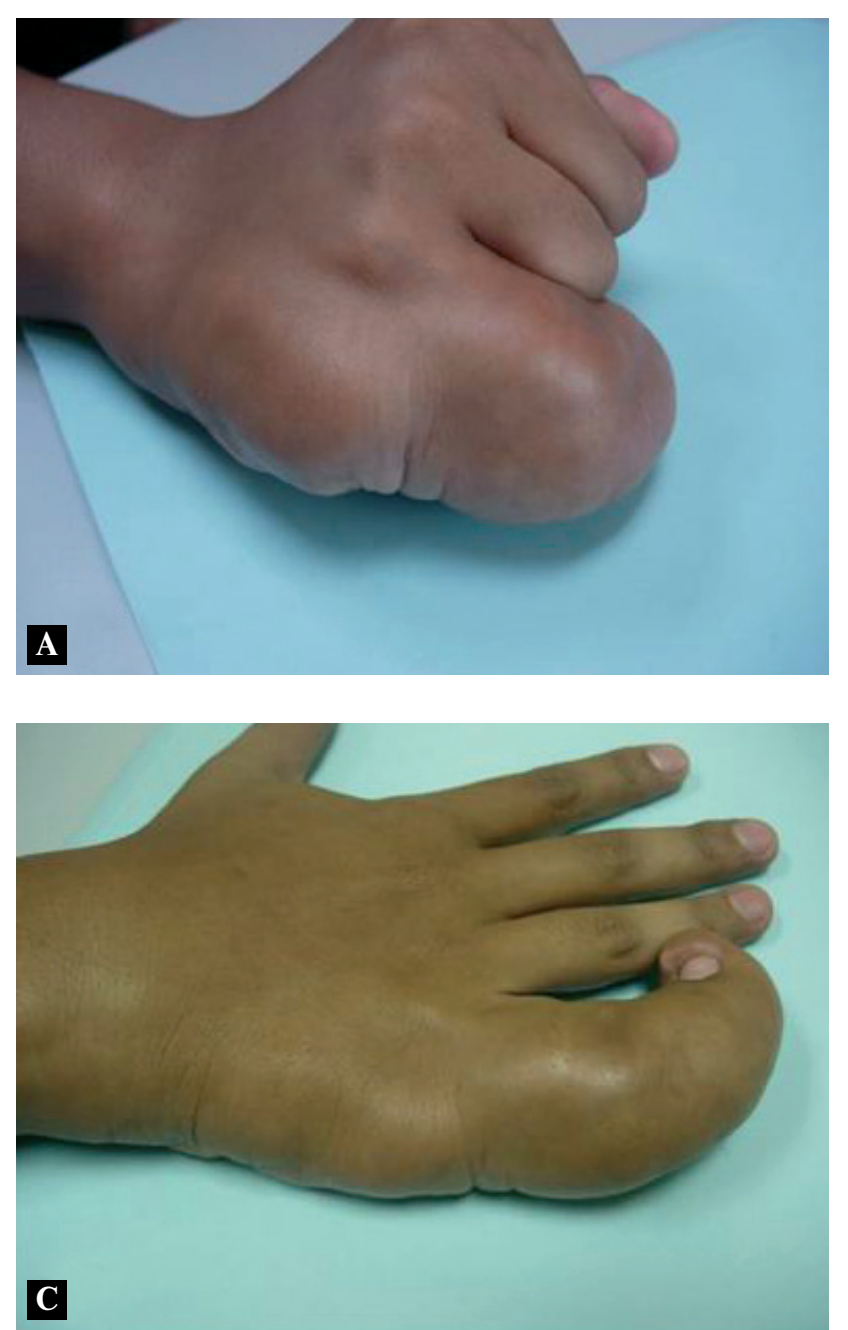

\section{Discussion}

PS is a rare congenital disorder. PS was first described by Cohen and Hayden in 1979 when they reported this disorder in two patients (3). Later on, a German Pediatrician, Hans-Rudolf Wiedemann named it proteus syndrome, after the Greek God Proteus "the polymorphous" because of its variable manifestation (11). PS is a complex hamartomatous disorder which is characterized by local overgrowth (macrodactyly or hemihypertrophy), subcutaneous tumours and various bone, cutaneous and/or vascular anomalies (4). Perhaps these are important features to look for while diagnosing PS. The diagnosis of PS is difficult because the clinical features of PS often overlaps with with other overgrowth or hamartomatous disorders (7). Differential diagnosis includes various conditions with overgrowth of tissues like bone, skin and central nervous system. Macrodactyly may be a common feature in other diseases also. PS should be differentiated from other conditions like hamartoses, skin
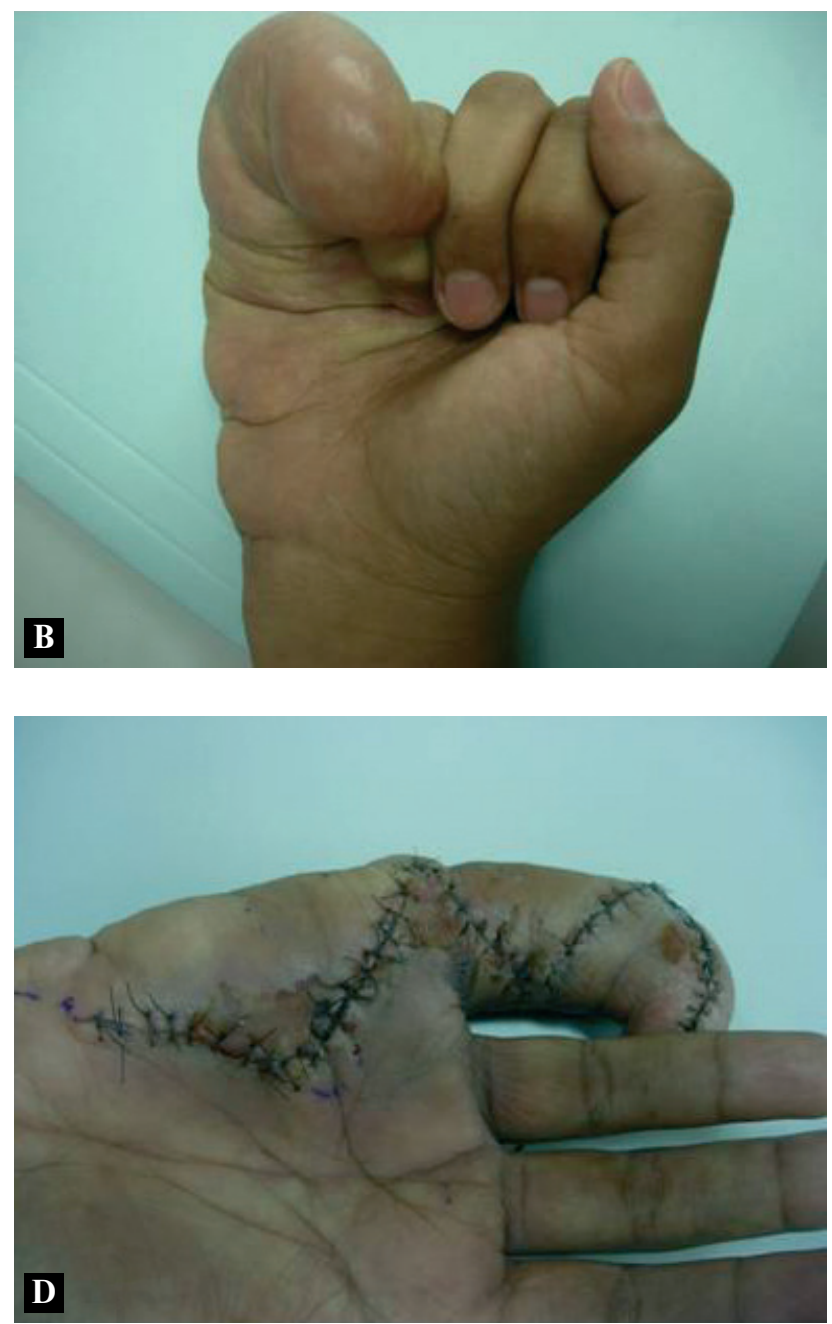

Fig. 1A,B,C: Photograph showing an overgrown right little finger involving the base of the hypothenar eminence. Fig. 1D: Photograph showing post debulking surgery of the right little finger. 

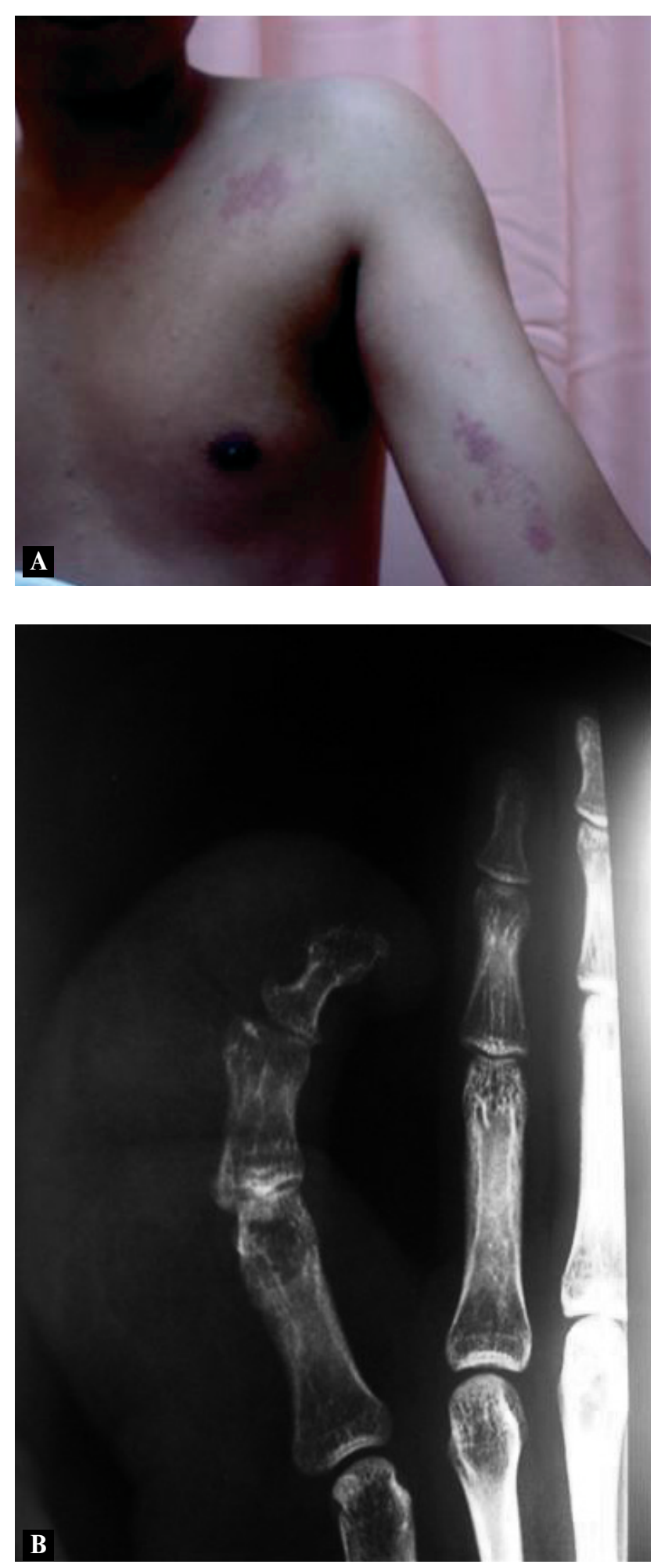

Fig. 2A: Photograph showing subcutaneous purplish pigmentation over the left upper chest and arm.

Fig. 2B: Radiograph the of right little finger with a slightly delta middle phalanx deviating radially. The bulky soft tissue silhouette can be clearly seen. lesions, vascular anomalies and neurocutaneous diseases. To differentiate it from other overgrowth conditions, it should be borne in mind that the overgrowth in PS is usually asymmetric in nature.

Skin involvement in PS include cerebriform connective tissue nevi, epidermal nevi, vascular malformations, lipohypoplasia and dermal hypoplasia $(8,10)$ and these symptoms may be observed in other disorders thereby complicating the diagnosis. In the present case, the patient presented with subcutaneous purplish pigmentation over his left upper chest and arm.

The disorder primarily manifests as postnatal overgrowth, with irregular, distorting and progressive overgrowth that can include many tissues like bone, connective tissue and fat are the most commonly involved tissues. Overgrowth of the central nervous system, spleen, thymus, colon and other tissues were reported. Manifestations of overgrowth are almost always asymmetric. The presentation of
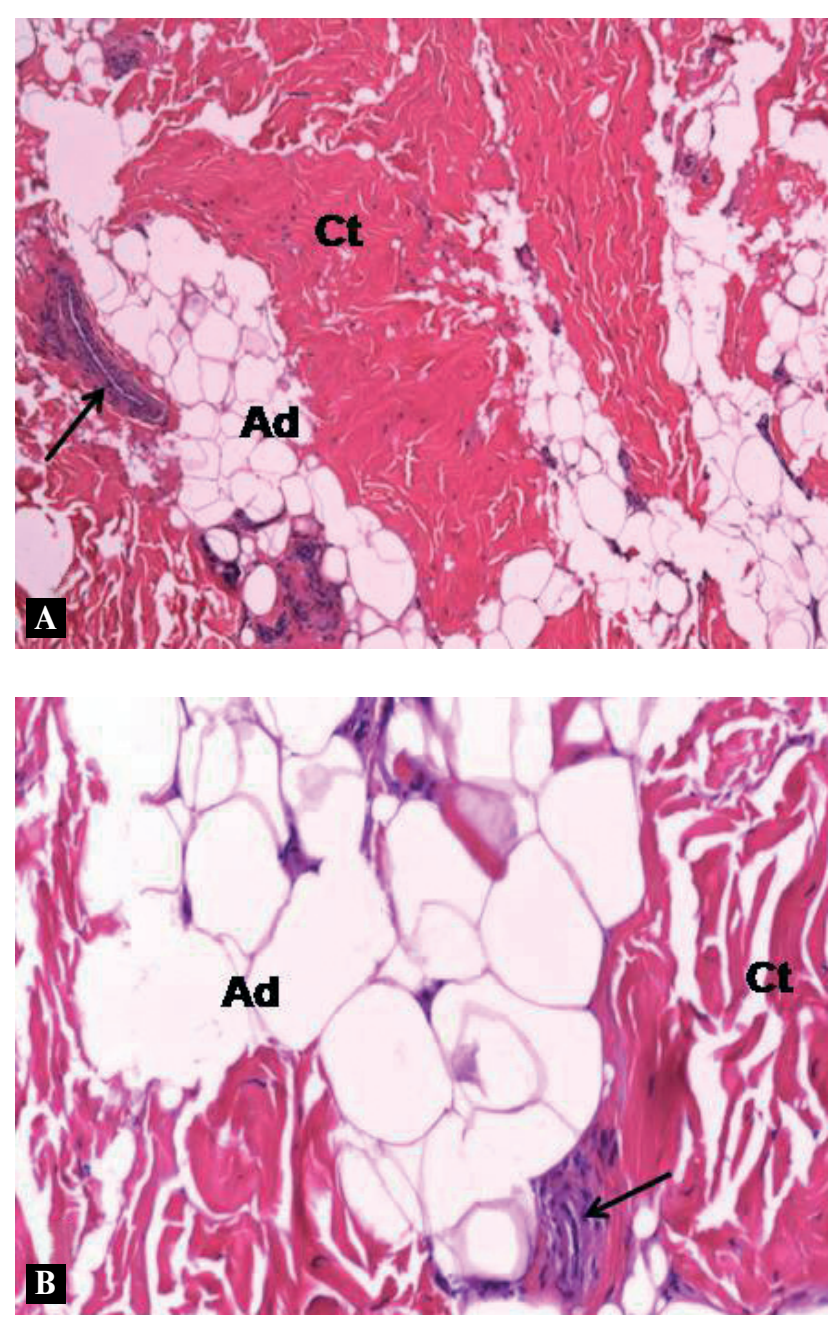

Fig. 3A,B: Photomicrograph of the histopathological slide, showing adipose tissue (Ad), connective tissue $(\mathrm{Ct})$. The neurovascular bundle is shown with arrow. 
overgrowth in a symmetric pattern (e.g. overgrowth of both hands and both feet) should lead one to doubt the diagnosis of PS. However, not all patients with asymmetric overgrowth, vascular malformations and skin lesions have PS (11).

A rating scale was proposed by Hotamisligil based on the most frequent and most characteristic features of fifty five cases of Proteus syndrome (5). A certain feature was given a certain point eg. macrodactyly and/or hemihypertrophy (5 points), macrocephaly (2.5 points), verrucous epidermal nevus ( 3 points), skin thickening ( 4 points), lipomas and subcutaneous tumours (4 points) and other minor abnomalities ( 1 point). Thirteen or more points are needed to establish the proper diagnosis of Proteus syndrome. According to the mentioned rating scale, the total of points of our case was 13: hemihypertrophy (5 points), skin thickening ( 4 points) and subcutaneous tumours (4 points). Our patient does have an epidermal nevus but it was not verrucous in nature.

Bisecker proposed a more recent diagnostic criteria for PS (1). It has a category for general criteria (mosaic distribution, progressive and sporadic) and specifc criterias. Our patient possessed all three general criteria but partially for Category B (ie epidermal nevus and disproportionate overgrowth of only $1 \mathrm{limb}$ ). However, the complex and variable features of PS do not necessarily allow easy compartmentalization of these criterias. Cutaneous and extracutaneous abnormalities should be examined carefully with dermatologists playing an important role.

In the present case, histopathological examination of the fatty tissue revealed well formed lobules of mature adipocytes interspersed with fibrous connective tissue elements within which there were vessels and nerves, suggestive of overgrowth. Interestingly, recent studies have described that PS can result in deficient growth as well as overgrowth (6). The presence of pigmentation in our patient is in accordance with earlier research reports which described hyperpigmented and hypopigmented macules (6).
Dysregulation of fatty tissue is a hallmark of PS. Localized overgrowth of fatty tissue in areas that normally have such tissue and these fatty tissue overgrowths are not encapsulated. PS is challenging due to its rapid overgrowth.

Treatment involves tumour debulking or amputation. In the present case, we had successfully performed a debulking surgery. There is no consensus regarding absolute necessary time for surgical intervention. Overgrowth of multiple body tissues require a multidisciplinary approach involving various specialties for effective treatment of PS. Most common causes of death for patients with PS is deep venous thrombosis and pulmonary embolism, even in young children.

\section{References}

1. Biesecker LG, Happle R, Mulliken JB et al. Proteus syndrome: diagnostic criteria, differential diagnosis, and patient evaluation. Am J Med Genet 1999; 84 389-95.

2. Cohen MM Jr. Proteus Syndrome: an update. Am J Med Genet C Semin Med Genet. 2005; 137C(1): 38-52.

3. Cohen MM Jr, Hayden PW. A newly recognized hamartomatous syndrome. Birth Defects 1979; 15: 291-296.

4. Hoeger PH, Martinez A, Maerker J, Harper JI. Vascular anomalies in Proteus syndrome. Clin Exp Dermatol 2004; 29: 222-30.

5. Hotamisligil GS. Proteus syndrome and hamartoses with overgrowth Dysmorphol Clin Genet 1990; 4: 87

6. Turner JT, Cohen MM Jr, Biesecker LG. Reassessment of the Proteus syndrome literature: application of diagnostic criteria to published cases. Am J Med Genet 2004; 130A: 111-22.

7. Nguyen D, Turner JT, Olsen C, et al. Cutaneous manifestations of proteus syndrome: correlations with general clinical severity. Arch Dermatol 2004; 140: 947-53.

8. Raju RR, Hart WR, Magnuson DK, et al. Genital tract tumors in Proteus syndrome: report of a case of bilateral paraovarian endometrioid cystic tumors of borderline malignancy and review of the literature. Mod Pathol 2002; 15: 172-80.

9. Samlaska CP, Levin SW, James WD, Benson PM, Walker JC, Perlik PC. Proteus syndrome. Arch Dermatol 1989; 125: 1109-14.

10. Viljoen DL, Saxe N, Temple-Camp C. Cutaneous manifestations of the Proteus syndrome. Pediatr Dermatol 1988; 5: 14-21.

11. Wiedemann HR, Burgio GR, Aldenhoff $P$, et al. The Proteus syndrome: partial gigantism of the hand and /or feet, nevi, hemihypertrophy, subcutaneous tumours, macrocephaly or other skull anomalies and possible accelerated growth and visceral affections. Eur J Pediatr 1983; 140: 5-12.

Received: $16 / 05 / 2009$.

Accepted in revised form: 23/10/2010.

Corresponding author:

Shalimar Abdullah, Department of Orthopaedics, Faculty of Medicine, Universiti Kebangsaan Malaysia Medical Centre (UKMMC), Jalan Yaacob Latiff, Bandar Tun Razak, Cheras, 56000 Kuala Lumpur, Malaysia; e-mail: kelapa44@yahoo.com 